\title{
Territoires et identités en Péninsule indochinoise : les Akha et la montagne au Laos
}

\section{Marianne Blache}

\section{(2) OpenEdition}

1 Journals

Édition électronique

URL : http://journals.openedition.org/cdg/2830

DOI : $10.4000 /$ cdg. 2830

ISSN : 2107-7266

Éditeur

UMR 245 - CESSMA

Référence électronique

Marianne Blache, «Territoires et identités en Péninsule indochinoise : les Akha et la montagne au Laos », Carnets de géographes [En ligne], 2 | 2011, mis en ligne le 02 mars 2011, consulté le 07 mai 2019. URL : http://journals.openedition.org/cdg/2830; DOI : 10.4000/cdg.2830

Ce document a été généré automatiquement le 7 mai 2019.

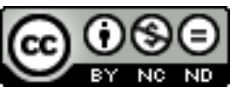

La revue Carnets de géographes est mise à disposition selon les termes de la Licence Creative Commons Attribution - Pas d'Utilisation Commerciale - Pas de Modification 4.0 International. 


\title{
Territoires et identités en Péninsule indochinoise : les Akha et la montagne au Laos
}

\author{
Marianne Blache
}

1 Cette thèse s'interroge sur les rapports entre le territoire, espace physique, organisé et approprié par un groupe humain, et l'identité de ce groupe humain. L'aire géographique qui a été choisie pour révéler les caractéristiques et les enjeux contemporains de ces rapports est la Péninsule indochinoise, et plus particulièrement le Laos.

Dans ce pays, à l'image de ce qu'on trouve dans une grande partie de l'Asie du Sud-Est continentale, s'opposent des plaines et vallées, organisées en casiers rizicoles, plus densément peuplées, à des montagnes vides, trouées d'essarts sur brûlis. La dichotomie entre plaines et montagnes n'est pas seulement physique et paysagère : alors que les basses terres sont peuplées de groupes ethniques relativement homogènes, d'origine linguistique thaï, les hauteurs présentent une véritable mosaïque ethnique, composée de groupes autochtones, de migrants venus du sud de la Chine au siècle dernier.

3 Le regard du géographe se trouve interpellé par une telle discontinuité, mais peu de travaux ont considéré ces deux types d'espaces comme pouvant être de véritables territoires, porteurs d'identité. Aussi, mes démarches ont abouti à une nouvelle lecture de l'organisation territoriale et des dynamiques de cette région du monde. La comparaison a été menée entre un groupe montagnard, la minorité ethnique Akha venue de Chine, et la majorité Lao-thaï installée dans les vallées et plaines du Laos.

\section{Situation de la zone enquêtée}

Dans un premier temps, la question a été de savoir si ces deux espaces, basses terres et montagnes, sont de véritables territoires pour les populations qui les habitent. Chez l'un comme chez l'autre peuple, tout un ensemble de référentiels spatiaux: sens de la pente pour les Akha, axe du fleuve pour les Lao, guident les configurations territoriales aux échelles de la maison, du village et du terroir. Le couple sauvage/domestique, ritualisé et 
concrétisé par différents moyens, se traduit par une porosité des limites, par des effets de seuils, qui induisent un rapport à l'environnement des habitants des hauteurs totalement distinct de celui des gens de plaine. À cette première échelle, celle de l'espace vécu villageois, le territoire, marqué culturellement, permet de reproduire une identité spatiale particulière, une territorialité dans laquelle on retrouve nettement les éléments montagnards chez les akha, et les éléments liés aux basses terres, comme le riz inondé, chez les Lao.

Dans un deuxième temps, il a fallu se demander si ces éléments de territorialisation se reproduisaient à une échelle plus vaste, de manière à former de véritables territoires identitaires. Après un détour par les catégorisations ethniques, il est montré que ce territoire identitaire est un ensemble complexe d'espaces vécus, pratiqués, construits dans le temps et représentés par un groupe. En analysant les dynamiques actuelles et passées de ces territoires, leurs caractéristiques ont pu être révélées. Ainsi, le territoire identitaire akha est un territoire- milieu, fluide et plastique : la composante montagnarde, forestière, accompagnée de faibles densités de population, et de la présence d'autres villages akha alentour, sont les conditions de reproduction de l'identité akha. La migration des villages, au sein de ce même milieu, ne transforme pas cette identité, elle en est constitutive.

6 À l'opposé, le territoire identitaire Lao-thaï est plus classiquement un territoire-lieu : l'identité des différents sous-groupes s'est formée en référence à un lieu précis. Ce lieu pourrait correspondre au concept de muang, mot polysémique signifiant en lao à la fois pays, royaume, ville, et région rurale organisée par cette même ville.

7 Aujourd'hui, l'identité territoriale des populations montagnardes est menacée par des déplacements massifs de la montagne vers les plaines, qui ont lieu sous le coup des politiques publiques, environnementales et foncières, menées au Laos.

8 Alors que ces deux types de territoires ont longtemps été juxtaposés, c'est sous une même autorité étatique qu'ils évoluent aujourd'hui : la colonisation a introduit des enveloppes territoriales nationales, fixées par des frontières modernes.

9 Aussi, le gouvernement autoritaire du Laos, tenu depuis toujours par la majorité culturelle lao-thaï, découvre les potentialités de la partie montagnarde de son territoire national. Cet espace, qui représente la majorité de sa surface, reste à intégrer : intégration politique et sociale de ses habitants, intégration économique d'une périphérie encore largement sous-exploitée.

Les représentations de l'espace montagnard par les acteurs des basses-terres sont en train de changer dans un contexte d'ouverture économique: la montagne peut devenir une ressource non négligeable, support d'activités minières, sylvicoles, hydroélectriques, et touristiques. Ces nouvelles problématiques donnent lieu à de nombreuses actions contradictoires de la part des autorités, révélant le rapport complexe entre les échelles administratives de ce pays, et les paradoxes du développement d'un pays pauvre.

11 L'étude de la territorialité et de la construction territoriale est d'abord passée par la compilation et la synthèse de données provenant d'un important corpus bibliographique, issu de travaux anthropologiques et historiques. Les représentations cartographiques et iconographiques produites par les différents acteurs du territoire ont également servi de base à l'étude des représentations spatiales.

12 Ensuite, le travail de terrain, en plusieurs séjours, a permis de connaître les espaces pratiqués et représentés des différentes populations, dans la province septentrionale de 
Luang Namtha. Six villages ont été choisis : quatre akha, dont deux déplacés en plaine, et deux lao-thaï. Un des villages akha a été enquêté de manière diachronique, avant et après son déplacement en plaine. L'enquête a employé deux méthodes principales :

- un questionnaire portant sur les déplacements annuels des individus, dans quatre villages, représentant un total de 71 ménages. Une base de données SIG a été construite à partir de ces données.

- le recueil de cartes mentales collectives et individuelles pour repérer les référents spatiaux des différents groupes.

Sinon, la caméra vidéo, utilisée comme carnet de notes, a permis la production d'un documentaire présenté lors de la soutenance.

\section{Discipline \\ Géographie \\ Directeur \\ Olivier Sevin \\ Université \\ Université Paris Sorbonne, Paris IV.}

Membres du jury de thèse, soutenue le 20 novembre 2010

- Christian Huetz de Lemps, professeur de géographie à l'Université Paris Sorbonne, président du jury.

- François Bart, professeur de géographie à l'Université, rapporteur.

- Manuelle Franck, professeur de géographie à l'INALCO, rapporteur.

- Bernard Moizo, directeur de recherche en socio-anthropologie à l'IRD.

- Olivier Sevin, professeur de géographie à l'Université Paris Sorbonne, directeur de thèse.

Contact de l'auteur

marianne.blache@wanadoo.fr

\section{INDEX}

Thèmes : Carnets de soutenances 